\title{
Cortical Brain Regions Associated with Color Processing: An FMRi Study
}

\author{
Inês Bramão $0^{1,3,4}$, Luís Faísca ${ }^{1}$, Christian Forkstam ${ }^{2,3,4}$, Alexandra Reis ${ }^{1,2}$ and Karl Magnus \\ Petersson* $*, 1,2,3,4$
}

\author{
${ }^{1}$ Cognitive Neuroscience Research Group, Deparmento de Psicologia, Faculdade de Ciências Humanas e Sociais, \& \\ Institute of Biotechnology \& Bioengineering/CBME, Universidade do Algarve, Faro, Portugal \\ ${ }^{2}$ Cognitive Neurophysiology Research Group, Stockholm Brain Institute, Karolinska Institutet, Stockholm, Sweden \\ ${ }^{3}$ Max Planck Institute for Psycholinguistics, Nijmegen, the Netherlands \\ ${ }^{4}$ Donders Institute for Brain, Cognition, and Behaviour, Centre for Neuroimaging, Radboud Universiteit Nijmegen, the \\ Netherlands
}

\begin{abstract}
To clarify whether the neural pathways concerning color processing are the same for natural objects, for artifacts objects and for non-objects we examined brain responses measured with functional magnetic resonance imaging (FMRI) during a covert naming task including the factors color (color $v s$. black\&white $(\mathrm{B} \& \mathrm{~W})$ ) and stimulus type (natural $v s$. artifacts $v s$. non-objects). Our results indicate that the superior parietal lobule and precuneus (BA 7) bilaterally, the right hippocampus and the right fusifom gyrus (V4) make part of a network responsible for color processing both for natural objects and artifacts, but not for non-objects. When color objects (both natural and artifacts) were contrasted with color non-objects we observed activations in the right parahippocampal gyrus (BA 35/36), the superior parietal lobule (BA 7) bilaterally, the left inferior middle temporal region (BA 20/21) and the inferior and superior frontal regions (BA 10/11/47). These additional activations suggest that colored objects recruit brain regions that are related to visual semantic information/retrieval and brain regions related to visuo-spatial processing. Overall, the results suggest that color information is an attribute that can improve object recognition (behavioral results) and activate a specific neural network related to visual semantic information that is more extensive than for $\mathrm{B} \& \mathrm{~W}$ objects during object recognition.
\end{abstract}

Keywords: FMRI, color information and processing, naming, natural objects, artifacts objects, non-objects.

\section{INTRODUCTION}

Traditionally, theories about object recognition have emphasized the role of shape information in higher-level vision $[1,2]$. More recently, data from behavioral studies, neuroimaging, and neuropsychological studies have suggested that surface features, such as color, also contribute to object recognition [for a review, 3]. However, the conditions under which surface color improves object recognition are not well understood. One general idea is that surface color improves the recognition of objects from natural categories, but not the recognition of artifact categories [4-6]. Humphreys and colleagues showed that objects from structurally similar categories, such as natural objects, take longer to identify than items from structurally dissimilar categories, such as artifacts, because the representations of structurally similar objects are more likely to be co-activated, therefore resulting in greater levels of competition within the object recognition system. Apparently, surface details such as color can help in resolving this competition [7-9]. Another potential reason that color information might help in recognizing natural

*Address correspondence to this author at the Max Planck Institute for Psycholinguistics, P.O.Box 310, NL-6500 AH Nijmegen, The Netherlands; Tel: +31 (0)24 3521319; Fax: +31 (0)24 3521213;

E-mail: karl-magnus.petersson@mpi.nl objects is color diagnosticity. Color diagnosticity means the degree to which a particular object is associated with a specific color. Several experiments have shown that visual recognition of diagnostic colored objects benefits from surface color information, whereas recognition of nondiagnostic colored objects does not [10-12]. Typically, natural objects are more strongly associated with a specific color than artifacts. For example, a strawberry - a diagnostic colored object - is clearly associated with the color red, whereas a comb - a non-diagnostic colored object - is not strongly associated with any specific color when using color as a cue for object identification [6]. Nagai and Yokosawa [10] studied the interaction between color diagnosticity and semantic category in order to determine whether surface color helps in the recognition of diagnostic colored objects independently from their semantic category. In a classification experiment, surface color improved the recognition of diagnostic colored objects independently from their category, supporting the hypothesis that color diagnosticity is an important cue for object recognition.

In this study, we used functional magnetic resonance imaging (FMRI) to investigate whether color information plays a different role in the recognition of natural objects compared to artifacts. We examined FMRI responses during a naming task that involved natural objects and artifacts presented in both color and black \& white $(\mathrm{B} \& \mathrm{~W})$. The color diagnosticity was kept constant between the two categories 
[13]. If color information plays a different role in the recognition of artifacts compared to natural objects, then different brain regions should be engaged during the naming of colored objects from different categories.

The neural correlates of color processing have been thoroughly investigated. Previous functional neuroimaging studies have associated area V4, located within the fusiform gyrus, as a centre of color perception in the human brain [1420]. At the neuroanatomical level, area V4 is involved in color constancy operations $[14,21]$, color ordering tasks [22], object color recognition [23-25], conscious color perception [26], color imagery [27] and color knowledge [28]. Whereas V4 has been associated with color perception, the left inferior temporal gyrus has been described as the site of stored information about color [23, 29]. For example, Chao and Martin [23] argue that the cortical areas that subserve color knowledge are distinct from the cortical areas that subserve color perception.

With regard to colored object recognition, Zeki and Marini [25] found that both naturally and unnaturally colored objects activated a pathway extending from the posterior occipital V1 to the posterior fusiform V4. In addition to the posterior parts of the fusiform gyrus, naturally colored objects activated the medial temporal lobe and the ventrolateral prefrontal cortex. These results suggest three broad cortical stages for color processing. The first stage is based in V1, and possibly V2, and is mainly concerned with registering the presence and intensity of different wavelengths and wavelength differences. The second stage, supported by V4, involves automatic color constancy operations and is independent from memory operations, perceptual judgment or learning. The third and final stage, based in the inferior temporal and frontal cortices, processes information for naturally colored objects and involves memory, judgment and learning [25].

One question of interest in this context is the role of surface color when color is a property of an object compared to when color is part of an abstract composition or a nonobject. According to the three-stage model for color processing outlined by Zeki and Marini [25], colored natural objects and artifacts should engage brain regions involved in the third stage of color processing, whereas colored nonobjects should only engage brain regions involved in the first two stages. To address this issue, in addition to natural objects and artifacts, we included non-objects presented in color and in $\mathrm{B} \& \mathrm{~W}$.

In summary, in the present FMRI study, we investigated whether the neural correlates of color information are the same for natural objects and artifacts. We also assessed the brain regions that are specific for color when color is a property of a recognizable object compared to when color is part of an unrecognizable composition. To address these issues, we examined FMRI responses in a silent naming task with two factors: color (color vs. B\&W) and stimulus type (natural objects $v s$. artifacts $v s$. non-objects). We expected to find fusiform gyrus (V4) activation in the color vs. B\&W stimuli (for both objects and non-objects), confirming that the fusiform gyrus is the brain center for color perception. Additionally, we hypothesized that colored natural objects and artifacts would engage brain regions involved with color knowledge information and retrieval (inferior temporal and frontal activation) to a greater degree compared with $\mathrm{B} \& \mathrm{~W}$ natural objects and artifacts.

\section{METHODS}

\section{Participants}

Twenty right-handed Portuguese native speakers [mean age $( \pm$ std $)=22 \pm 4$ years; mean years of education $( \pm$ std $)=$ $14 \pm 1$ years; 5 men and 15 women] with normal or corrected-to-normal vision participated in the study. All subjects completed health questionnaires prior to scanning, and none reported a history of head injury or other neurological or psychiatric problems. All subjects read and signed an informed consent form describing the procedures according to the Declaration of Helsinki. The study was approved by the local ethics committee.

\section{Stimulus Material}

We selected 56 drawings from the Snodgrass and Vanderwart set [30]. Twenty-eight objects were from natural categories (animals and fruits) and twenty-eight were artifacts (tools and vehicles; see Table 1). We also constructed 28 matching non-objects (constructed with the Paint-software and approximately matched for visual complexity). The non-objects were scrambled lines and shapes without any obvious conventional meaning. All images were presented both in color and B\&W. The color version was selected from the set of Rossion and Pourtois [13] and the B\&W version was selected from the grey-scale set of Rossion and Pourtois [13]. We opted for the grey-scale version and not the original $\mathrm{B} \& \mathrm{~W}$ version from the Snodgrass and Vanderwart [30] set in order to keep the luminance and brightness constant over the color and B\&W conditions (Fig. 1). All 56 images were classified according to familiarity based on norms for the Portuguese population [31], color diagnosticity based on Rossion and Pourtois [13], and visual complexity based on the original work of Snodgrass and Vanderwart [30]. There was no significant difference between stimulus types on these variables $(\mathrm{P}>$ $0.10)$. In addition, the natural and artifact stimuli were matched in terms of syllabic length $(P>0.20)$. Stimuli luminance, measured using Adobe Photoshop 7.0, of the natural objects, artifacts and non-objects (color and B\&W versions) was similar (overall, Kruskall-Wallis ANOVA $\mathrm{H}<$ 1.6, $\mathrm{P}>0.50)$.

\section{Experimental Procedures}

The stimuli were presented in a blocked design. The twenty-eight stimuli from each condition were distributed over four blocks (6 conditions x 4 blocks; seven objects in each block) resulting in twenty-four blocks. Four of each condition were allocated to two different sets (each set was composed of 84 stimuli grouped into two blocks for each condition -2 blocks $\times 6$ conditions $\times 7$ stimuli). Two additional sets were constructed by changing the presentation order of the blocks in the two original sets. In each experimental set, we also included four blocks of seven baseline events, consisting of a visual fixation cross. For each subject, four sets were presented in four consecutive FMRI sessions. Altogether, 112 objects were presented to each subject per FMRI session, which included the seven experimental conditions: $\mathrm{CN}$ - colored natural objects; BWN - B\&W natural objects; CA - colored artifacts; BWA - 
Table 1. Stimuli Used in Experiment

\begin{tabular}{|c|c|}
\hline Natural Objects & Artifacts Objects \\
\hline Alligator & Accordion \\
\hline Ant & Airplane \\
\hline Apple & Anchor \\
\hline Bear & Barrel \\
\hline Butterfly & Basket \\
\hline Chicken & Bell \\
\hline Cow & Belt \\
\hline Dog & Boot \\
\hline Duck & Bus \\
\hline Fox & Car \\
\hline Gorilla & Cigarette \\
\hline Grapes & Drum \\
\hline Horse & Flute \\
\hline Lemon & Fork \\
\hline Monkey & Glasses \\
\hline Mushroom & Gun \\
\hline Onion & Hammer \\
\hline Pear & Harp \\
\hline Pepper & Key \\
\hline Pig & Nail \\
\hline Potato & Needle \\
\hline Rabbit & Nut \\
\hline Rooster & Pencil \\
\hline Seal & Piano \\
\hline Squirrel & Scissors \\
\hline Strawberry & Shoe \\
\hline Tiger & Spoon \\
\hline Turtle & Thimble \\
\hline
\end{tabular}

$\mathrm{B} \& \mathrm{~W}$ artifacts; $\mathrm{CNO}$ - colored non-objects; BWNO - B\&W non-objects; and finally, VF - visual fixation, which served as a baseline condition. Each subject saw each object twice per condition during the experiment, but never in the same FMRI session. Each block lasted 19.6 seconds, and each stimulus was presented for 2.8 seconds (Fig. 2).

In four separate scanning sessions, with session order counterbalanced across subjects, subjects were asked to attentively view the picture and silently name each object, in a covert naming task. Each of the four FMRI sessions lasted 6 minutes. Subjects were also asked to silently repeat "tantan" for the non-objects and for the visual fixation cross in order to encourage attention to the stimuli without attaching
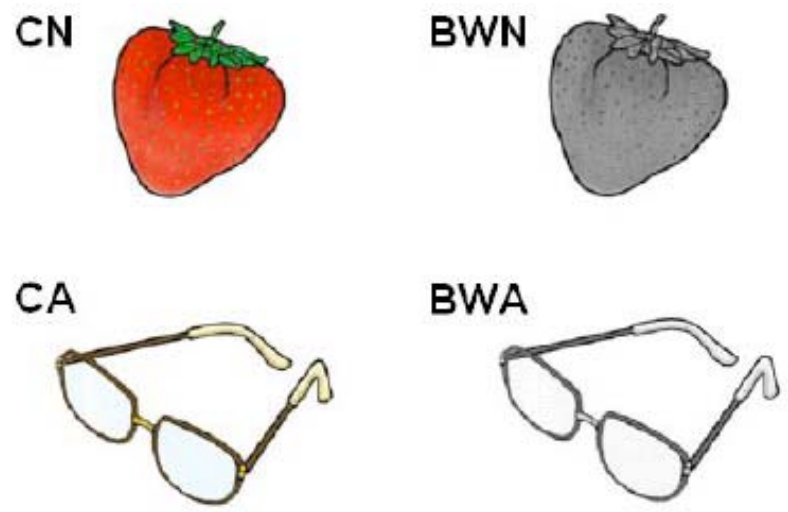

CNO

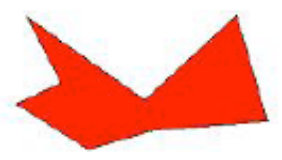

BWNO

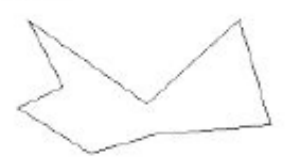

Fig. (1). Example of the stimuli used in the experiment. $\mathrm{CN}$ - color natural objects; BWN - B\&W natural objects; CA - color artifacts objects; BWA - B\&W artifacts objects; $\mathrm{CNO}$ - colored nonobjects; BWNO - B\&W non-objects.

a particular verbal label. Subjects viewed the stimuli via a mirror mounted on a head-coil (the visual angle for the stimulus presentation was approximately 8 degrees). Prior to the FMRI experiment, subjects performed an object naming task in order to familiarize themselves with the objects and for the acquisition of behavioral naming data. The verbal responses and naming times were registered for subsequent behavioral analysis. Voice detection equipment was used to register response times between the onset of the stimulus display and that of the response. The same presentation paradigm was used for the object naming task as for the FMRI experiment. The Presentation 0.7 Software (nbs.neuro-bs.com/presentation) was used to display the stimuli on a computer screen (HP Laptop with 15" screen) and to register the response times.

\section{MRI Data Acquisition}

We acquired whole head T2*-weighted EPI-BOLD MRI data with a Philips $1.5 \mathrm{~T}$ Intera scanner using a sequential slice acquisition sequence $\left(\mathrm{TR}=2.46 \mathrm{~s}, \mathrm{TE}=40 \mathrm{~ms}, 90^{\circ}\right.$ flip-angle, 29 axial slices, slice-matrix size $=64$ x 64, slice thickness $=3 \mathrm{~mm}$ with a slice gap $=0.4$, field of view $=220$ $\mathrm{mm}$, isotropic voxel size $=3.4 \times 3.4 \times 3.4 \mathrm{~mm}^{3}$ ). Following the experimental session, high-resolution structural images were acquired using a T1-weighted 3D TFE $(\mathrm{TE}=3.93 \mathrm{~ms}$, $10^{\circ}$ flip-angle, slice-matrix size $=256 \times 256$, field of view $=$ $256 \mathrm{~mm}, 200$ axial slices, slice thickness $=1.0 \mathrm{~mm}$, isotropic voxel-size $=1 \times 1 \times 1 \mathrm{~mm}^{3}$ ).

\section{MRI Data Analysis}

Image pre-processing and statistical analysis was performed using SPM5 (www.fil.ion.ucl.ac.uk/spm) implemented in MatLab (Mathworks, Sherborn, MA). The functional EPI-BOLD images were realigned and slice-time corrected, and the subject-mean functional MR images were 


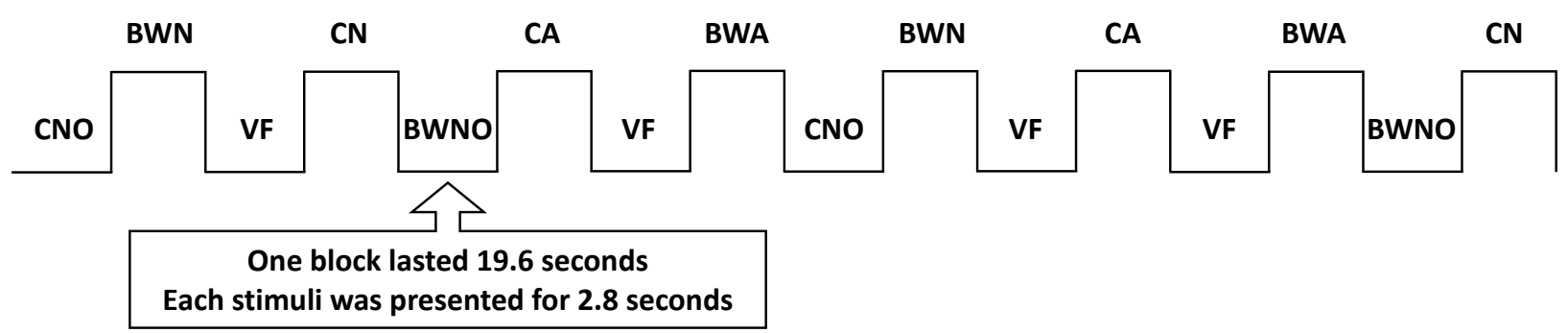

Fig. (2). Schematic representation of the experimental design for one FMRI session. CN - color natural objects, BWN - B\&W natural objects, CA - color artifacts objects, BWA - B\&W artifacts objects, CNO - colored non-objects, BWNO - B\&W non-objects, VF - visual fixation. Each block lasted 19.6 seconds and each stimulus was presented for 2.8 seconds.

co-registered with the corresponding structural MR images. These were subsequently anatomically normalized. The normalization transformations were generated from the structural MR images and applied to the functional MR images. The functional EPI-BOLD images were transformed into an approximate Talairach space [32] as defined by the SPM5 template and spatially filtered with an isotropic 3D spatial Gaussian kernel (FWHM $=10 \mathrm{~mm})$. The FMRI data were statistically analyzed using the general linear model and statistical parametric mapping [33]. At the first level, single-subject fixed effect analyses were conducted. The linear model included one box-car regressor for each of the $\mathrm{CN}, \mathrm{BWN}, \mathrm{CA}, \mathrm{BWA}, \mathrm{CNO}, \mathrm{BWNO}$ and VF conditions. We temporally convolved these explanatory variables with the canonical hemodynamic response function provided by SPM5. In addition, we also included realignment parameters to account for movement-related variability. The data were high-pass filtered (128 s) to account for various lowfrequency effects. For the second-level random effect analysis, we generated single-subject contrast images for the $\mathrm{CN}, \mathrm{BWN}, \mathrm{CA}, \mathrm{BWA}, \mathrm{CNO}$ and $\mathrm{BWNO}$ conditions relative to $\mathrm{VF}$.

We analyzed the contrast images in a two-way repeated measures ANOVA with the following factors: color type (color vs. B\&W) and stimulus type (natural objects $v s$. artifacts $v s$. non-objects). We analyzed the natural objects and artifacts together because there was no significant difference between these conditions, whether in color or $\mathrm{B} \& \mathrm{~W}$. Also, there was no significant difference in $\mathrm{CN} v s$. BWN or CA $v s$. BWA (overall, $P>0.3$ ). Thus, we generated single-subject contrasts for the colored objects $-\mathrm{CO}(=\mathrm{CN}+$ $\mathrm{CA})$ and the B\&W objects - BWO (= BWN + BWA). We analyzed these collapsed contrasts in a two-way repeated measures ANOVA with the following factors: color type (color $v s . \mathrm{B} \& \mathrm{~W}$ ) and stimulus type (objects $v s$. non-objects). Statistical inference was based on the cluster-size statistic from the relevant SPM[T] volumes. In a whole brain search, the results from the random effects analyses were initially threshold at with $\mathrm{P}<0.005$ (uncorrected) and only significant clusters at $\mathrm{P}<0.05$ (family-wise error (FWE) corrected for multiple non-independent comparisons) are reported [34]. All local maxima within significant clusters were subsequently reported with $\mathrm{P}$-values corrected for multiple non-independent comparisons based on the false discovery rate [FDR, 35]. SPM[T] volumes were generated to investigate the effects of color and stimulus type. Finally, we applied a small volume correction (SVC, $5 \mathrm{~mm}$ radius) to regions typically involved in color perception: the fusiform gyrus (V4) $([ \pm 28,-62,-20])$ and the hippocampus ([ \pm 36 , $10,-20])$ bilaterally $[23,25]$ and in a region in the left temporal gyrus previously described as the site of stored information about colored objects ([-56, -40, -14]) [23, 29]. All reported data are from the second-level random effect analyses. For portability of the results, we used the Talairach nomenclature [32] with the original SPM coordinates in the tables.

\section{RESULTS}

\section{Behavioral Results}

Subjects were able to correctly name all stimuli. Overall, the number of naming errors was small $(<2 \%)$, so we analyzed the naming times for the correct responses with latencies within 2.5 standard deviations from the mean for each subject and condition. Excessively long or short naming latencies were excluded from further analysis because these are likely due to lapses of attention/concentration and anticipatory responses, respectively. No-response trials and misregistered responses (software failure and responses anticipated by subject vocalizations other than the naming responses) were also excluded. In total, approximately $11 \%$ of the trials were excluded (3.8\% due to lapses of attention or concentration, $1.6 \%$ due to anticipatory responses, $1.5 \%$ due to incorrect responses, $0.1 \%$ due to non-answers, $3.8 \%$ due to misregistered responses). The naming times were analyzed with a repeated-measures ANOVA considering the following within factors: presentation version (color $v s$. $\mathrm{B} \& \mathrm{~W}$ ) and semantic category (natural objects $v s$. artifacts). The results showed a significant presentation version effect $\left[F(1,19)=30.6 ; \eta^{2}=0.62 ; P<0.001\right]-$ subjects were faster at naming color compared to $\mathrm{B} \& \mathrm{~W}$ objects. The semantic category effect $\left[\mathrm{F}(1,19)=2.8 ; \eta^{2}=0.13 ; \mathrm{P}=0.13\right]$ and the interaction between presentation version and category $\left[F(1,19)=0.10 ; \eta^{2}=0.005 ; P=0.76\right]$ were not significant (Fig. 3).

\section{FMRI Results}

\section{Color and B\&W Effects}

The contrast between color vs. B\&W stimuli (for both objects and non-objects) did not result in any significant activation, nor did the contrast between color non-objects $v s$. B\&W non-objects. However, the contrast between colored 


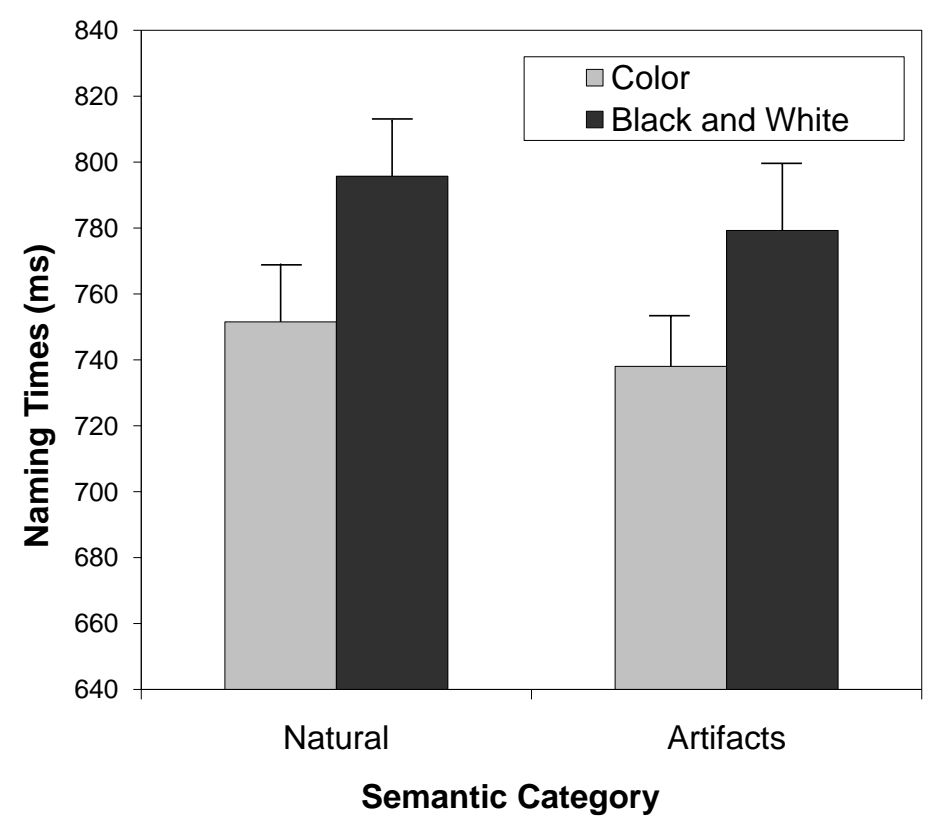

Fig. (3). Two-way interaction $\left[F(1,19)=2.8 ; \eta^{2}=0.13 ; P=0.13\right]$ between presentation version and semantic category on naming times.

objects $v s$. B\&W objects (Table 2) showed a significant cluster $(\mathrm{P}=0.006$, FWE corrected) that encompassed the superior parietal region and precuneus (BA 7) bilaterally. To further investigate the regional effects related to color processing, we used a regions-of-interest (ROI) approach in combination with small volume correction (SVC) for the family-wise error rate. We selected regions of interest based on previous investigations that studied color effect in object recognition [23, 25, 29]. These regions included the right/left fusiform gyrus (V4), the right/left hippocampus and the left inferior temporal gyrus. We investigated these regions in the following contrasts: 1) color $v s$. $\mathrm{B} \& \mathrm{~W}$ stimuli; 2) color $v s$. $\mathrm{B} \& \mathrm{~W}$ objects; and 3) color $v s$. B\&W non-objects. We found significant activations (Table $\mathbf{3}$ and Fig. 4) for color $v s$. $\mathrm{B} \& \mathrm{~W}(\mathrm{P}=0.032, \mathrm{SVC}$ corrected $)$ in the right hippocampus and for color $v s$. B\&W objects in the right fusiform gyrus (V4; $\mathrm{P}=0.011, \mathrm{SVC}$ corrected), right hippocampus $(\mathrm{P}=$ 0.033 , SVC corrected) and left temporal inferior gyrus $(\mathrm{P}=$ $0.037, \mathrm{SVC}$ corrected). The contrast between color $v s$. B\&W non-objects yielded no additional effects.

\section{Color and B\&W Object Recognition}

The contrast between object $v s$. non-object stimuli resulted in two clusters of significant brain activation $(\mathrm{P}<$ 0.001, FWE corrected). These included the posterior occipital regions (BA 18/19), the fusiform gyrus (BA 19/37), and the inferior temporal lobe (BA 20) bilaterally (Table 4 and Fig. 5). The contrast between colored objects vs. color non-objects (Table $\mathbf{4}$ and Fig. 5) resulted in additional activations in the right parahippocampal gyrus (BA 35/36), the inferior-superior parietal lobule (BA 7/39/40) bilaterally, and in the left inferior-middle temporal region (BA 20/21). In addition, frontal regions $(\mathrm{P}=0.006, \mathrm{FWE}$ corrected $)$ were also significantly activated in colored objects $v s$. colored non-objects, including the left anterior-inferior frontal region (BA 10/47) and the left superior frontal region (BA 10). The contrast between $\mathrm{B} \& \mathrm{~W}$ objects $v s$. $\mathrm{B} \& \mathrm{~W}$ non-objects activated similar brain regions as observed in objects $v s$. non-objects, however the activation pattern was more restricted and primarily observed in posterior brain regions (right: $\mathrm{P}=0.021$, FWE corrected; left: $\mathrm{P}=0.009$, FWE corrected; Table 4 and Fig. 5).

\section{DISCUSSION}

In this FMRI study, we aimed to clarify whether the neural substrates related to color information are the same when color is a property of a recognizable object, namely natural objects and artifacts, compared to when color is a property of an unrecognizable object, such as abstract compositions.

\section{Color Effects on Objects and Non-Objects}

According to the three cortical stages model for color processing proposed by Zeki and Marini [25], we expected that color information presented in recognizable objects would activate the V4 area as well as brain areas involved in memory, classification, and learning operations. Our results show that color compared to B\&W objects activated the right V4 area. In addition, we also observed brain activations in regions that are typically associated with color perception, the right hippocampus and superior parietal/precuneus region, corroborating previous findings $[14,18,20,23,25$, 27].

To better understand the role of color information in the recognition of familiar objects, we explored brain activation during the processing of colored objects and colored nonobjects. In general, object naming activated brain regions that extended from the occipital to the inferior temporal regions, including fusiform activation, consistent with earlier neuroimaging studies on object recognition [36-42]. Additionally, colored objects compared to colored nonobjects activated an extensive network of brain regions including the left inferior temporal gyrus, right 
Table 2. Color Objects and B\&W Objects

\begin{tabular}{|l|c|c|c|}
\hline \multicolumn{1}{|c|}{ Region } & Cluster Level & \multicolumn{2}{c|}{ Coordinates } \\
\cline { 2 - 5 } & PFW & x & \\
\hline \hline Color Objects versus B\&W Objects & & & \\
\hline Right superior parietal (BA 7) & 0.006 & 16 & -60 \\
\hline Left superior parietal (BA 7) & & -26 & -64 \\
\hline Right precuneus (BA 7) & & 10 & -48 \\
\hline Left precuneus (BA 7) & & -8 & 64 \\
\hline
\end{tabular}

SPM [T], Clusters significant at $P<0.05$ corrected for multiple non-independent comparisons are reported $\left(\mathrm{P}_{\mathrm{FWE}}\right)$. Local maxima within the clusters are reported. Coordinated are the original SPM $\mathrm{x}, \mathrm{y}, \mathrm{z}$ in millimeters of the MNI space.

Table 3. Small Volume Corrections in SPM [T]

\begin{tabular}{|c|c|c|c|c|c|}
\hline \multirow{2}{*}{ Region } & \multicolumn{2}{|c|}{ Voxel Lexel } & \multicolumn{3}{|c|}{ Coordinates } \\
\hline & $Z$ & $\boldsymbol{P}_{F W E}$ & $x$ & $y$ & $z$ \\
\hline \multicolumn{6}{|l|}{ Color versus B\&W } \\
\hline Right Hippocampus & 2.68 & 0.032 & 36 & -10 & -24 \\
\hline \multicolumn{6}{|c|}{ Color Objects versus B\&W Objects } \\
\hline Right Fusiform (V4) & 3.08 & 0.011 & 30 & -66 & -18 \\
\hline Right Hippocampus & 2.67 & 0.033 & 36 & -14 & -22 \\
\hline Left Inferior Temporal Gyrus & 2.62 & 0.037 & -60 & -42 & -16 \\
\hline
\end{tabular}

SPM [T], threshold at $P<0.005$, non-corrected. $\mathrm{P}_{\mathrm{FWE}} \mathrm{SVC}$ corrected. Coordinates are the original SPM x, $\mathrm{y}, \mathrm{z}$ in millimeters of the MNI space.

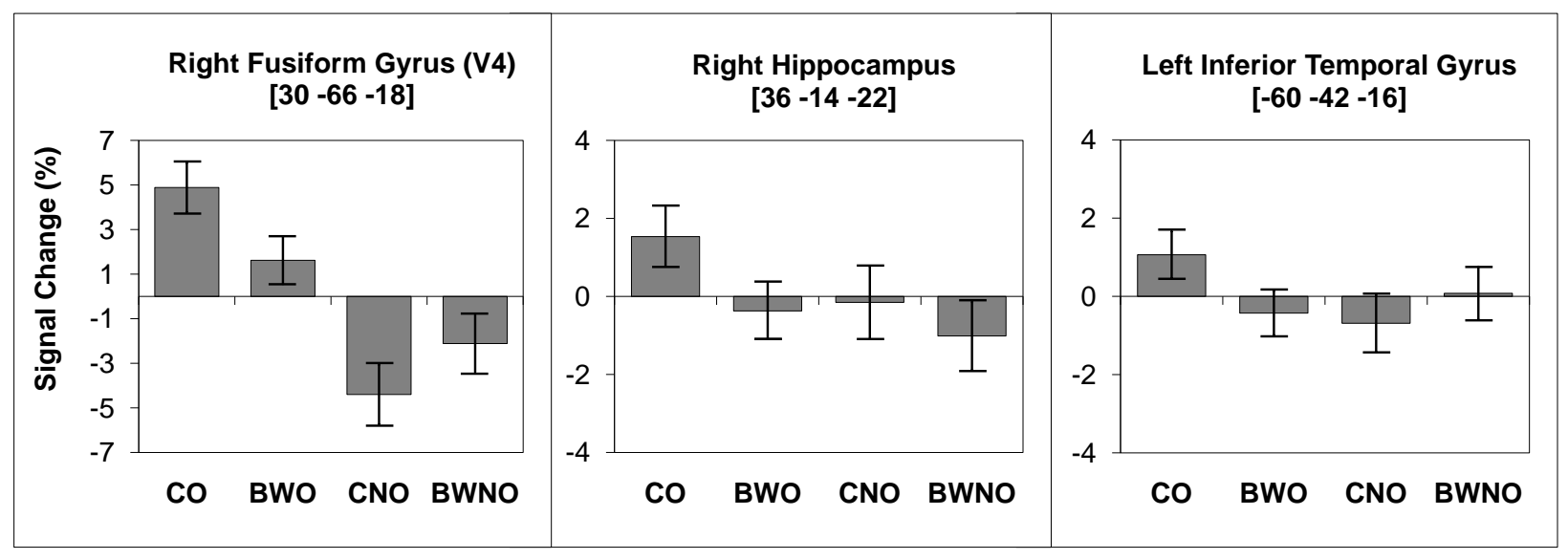

Fig. (4). BOLD signal change associated with color and $\mathrm{B} \& \mathrm{~W}$ objects $(\mathrm{CO}, \mathrm{BWO})$ and with color and $\mathrm{B} \& \mathrm{~W}$ non-objects $(\mathrm{CNO}, \mathrm{BWNO})$. 
Table 4. Objects Versus Non-Objects

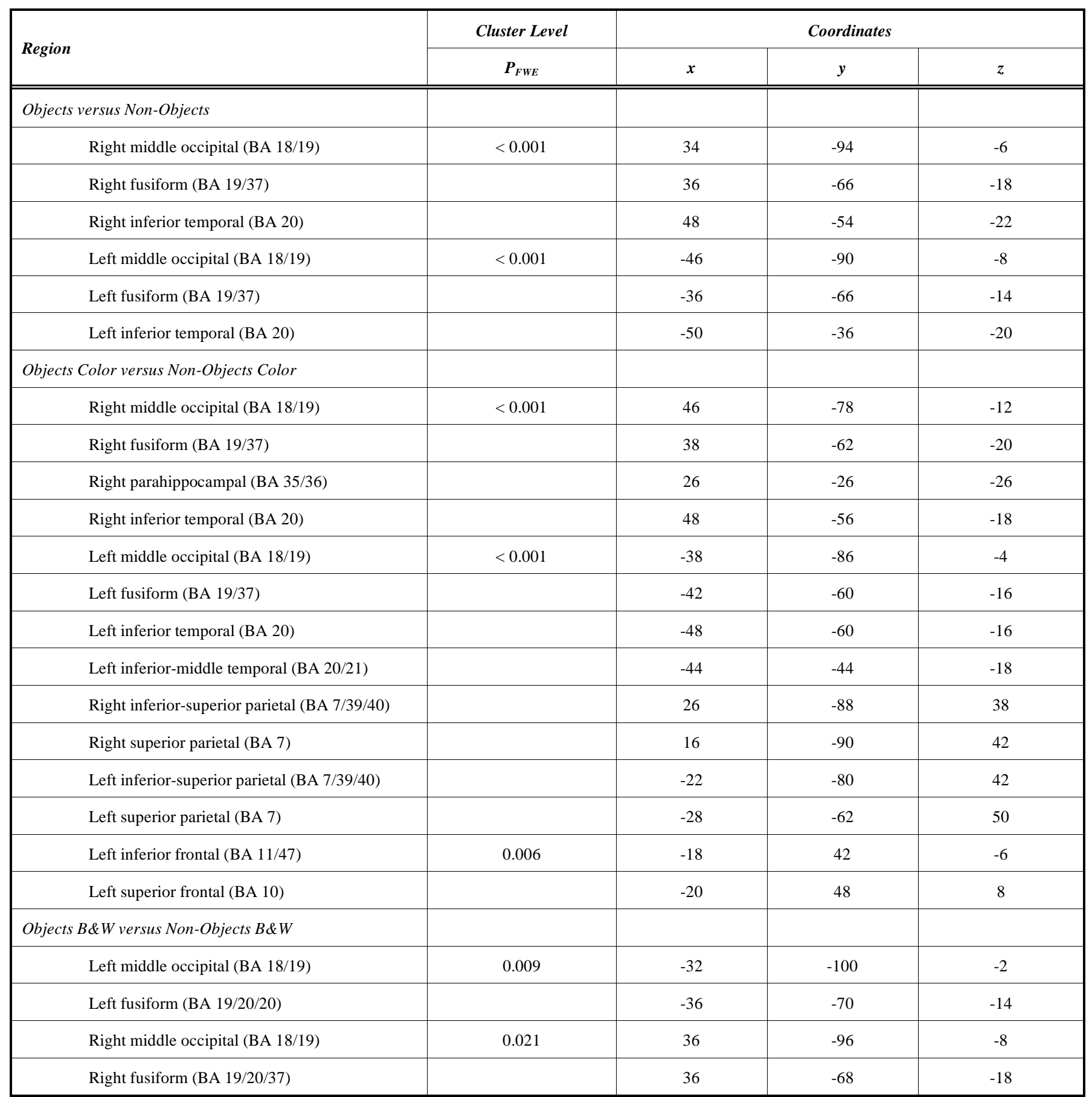

SPM [T], Clusters significant at P $<0.05$ corrected for multiple non-independent comparisons $\left(\mathrm{P}_{\mathrm{FWE}}\right)$ are reported. Local maxima within the clusters are reported. Coordinated are the original SPM $\mathrm{x}, \mathrm{y}, \mathrm{z}$ in millimeters of the MNI space.

parahippocampal gyrus, left inferior and superior parietal lobule, and left superior and anterior-inferior frontal regions. These activations were exclusive for colored objects and were not found when $\mathrm{B} \& \mathrm{~W}$ objects were contrasted against $\mathrm{B} \& \mathrm{~W}$ non-objects, suggesting that color plays an important role in accessing the semantic level during object naming processes, as initially suggested by Zeki and Marini [25]. We did not find any particular brain region that responded only to $\mathrm{B} \& \mathrm{~W}$ object naming, suggesting that the recognition of $\mathrm{B} \& \mathrm{~W}$ objects does not add a cognitive operation to the recognition of colored objects.
The temporal and frontal activations found during colored object naming suggest that color engages access to the semantic network that contains information/knowledge about the objects. Parahippocampal gyrus activation has been reported in post-recognition processes, such as visual and semantic analysis [43-45], and during the encoding and retrieval of color information $[46,47]$. It has been suggested that the inferior temporal gyrus stores information about colored objects [29, 48]. The frontal activations observed during colored object naming suggest that the recognition of a colored object engages a semantic network that is more 

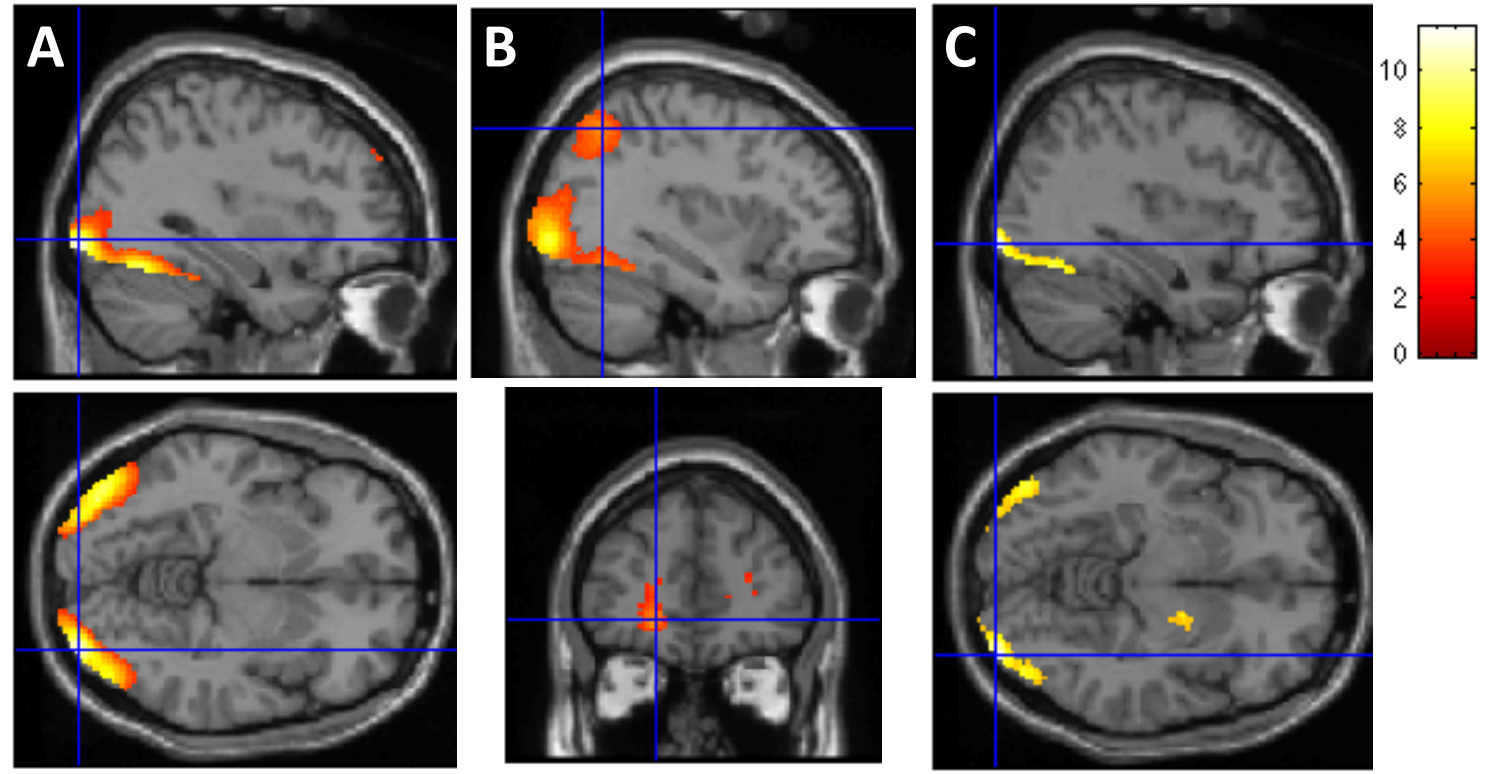

Fig. (5). A - Brain regions associated with objects compared to non-objects, B - Brain regions associated with color objects compared to color non-objects, $\mathbf{C}$ - Brain regions associated with $\mathrm{B} \& \mathrm{~W}$ objects compared to $\mathrm{B} \& \mathrm{~W}$ non-objects.

active in comparison to $\mathrm{B} \& \mathrm{~W}$ object recognition. Left inferior frontal activations have been reported during semantic knowledge tasks [40, 49-53]. On the other hand, the activations observed in the left inferior and superior parietal lobule might suggest that color is a feature that helps in the encoding of visuo-spatial properties of objects [54, 55]. An alternative explanation is that the activated parietal and frontal regions during the recognition of colored objects versus colored non-objects results from an increase in attention due to color information [56]. However, if this was the case, then we should have also seen this pattern of activation when colored non-objects were contrasted with B\&W non-objects.

Regarding the role of color information in the processing of non-objects, we expected that color information would engage V4. However, the contrast between colored versus $\mathrm{B} \& \mathrm{~W}$ non-objects did not yield an additional significant activation. The absence of V4 activation in the colored nonobject condition might be related to methodological issues such as a lack of sensitivity or to experimental design issues. Previous studies that reported V4 activation in response to abstract colored stimuli used transient on/off presentations of each stimulus at a rate of $1 \mathrm{~Hz}[18,25]$.

We should point out that there are other variables that could contribute to the pattern of the observed results. In every trial, subjects had to covertly utter the name of the recognized object or utter "tan-tan" for non-objects. In the case of non-objects, subjects knew from the start of the block that they would only have to utter "tan-tan" while the block was running, without any further processing. In contrast, for object blocks, subjects had to recognize and name every object. Consequently, producing the non-sense word "tantan" for all non-objects did not require the same level of complexity as retrieving lexical information for certain objects. This may therefore lead to condition-dependent biases in the associated attention state, lexical retrieval and covert naming. However, when we suggest that the colored objects (vs. colored non-objects) activated a more extensive brain network than the $\mathrm{B} \& \mathrm{~W}$ objects (vs. $\mathrm{B} \& \mathrm{~W}$ nonobjects), we are excluding the interference associated with attention state, lexical retrieval and covert naming because these effects are present in the contrast between both colored and B\&W objects $v s$. non-objects.

\section{Color Effects in Natural Objects and Artifacts}

Our results show that the brain regions responsible for color processing are the same when color is a property of natural objects and artifacts, suggesting that color information has the same role in the recognition of natural objects and artifacts. This result does not support the proposal of Humphreys and colleagues [4, 6] and suggests that previous behavioral differences reported in the processing of natural objects and artifacts might be due to color diagnosticity rather than to semantic category. When color diagnosticity is controlled, as in our study, no differences in the recognition of colored natural objects and artifacts were found in the FMRI or the behavioral results. Moreover, our results showed that the brain regions responsible for processing natural objects and artifacts are the same, both when the objects are presented in color and in B\&W. Several candidate regions have emerged as potential sites that may be strongly involved in natural object recognition, including the medial occipital, right occipitotemporal and left anterior temporal cortex [48, 57-59]. On the other hand, the fusiform gyrus, left precentral gyrus and left posterior middle temporal cortex have been reported as the sites that may be strongly involved in the recognition of artifacts compared with objects from other categories [48, 58]. Although category-specific brain activation patterns have been investigated in several neuroimaging studies, the results have not been consistent across studies. For example, Joseph [60] performed a meta-analysis of stereotactic coordinates to determine if category membership predicts patterns of brain region activation across different studies. The author found no more than $50 \%$ convergence for the recognition of both natural objects and artifacts in any brain region. 


\section{CONCLUSIONS}

Colored objects activate the inferior temporal, parahippocampal and inferior frontal brain regions, areas that are typically involved in visual semantic processing and retrieval. This suggests that the recognition of a colored object activates a semantic network in addition to the one that is active during the recognition of $\mathrm{B} \& \mathrm{~W}$ objects. The engagement of the semantic network when color is present in the objects led subjects to name colored objects more quickly than B\&W objects. These results suggest that color information can have an important role during the visual recognition process for familiar and recognizable objects (both natural objects and artifacts), facilitating semantic retrieval. On the other hand, color information present in non-objects does not activate any brain region involved in the recognition process itself, but only engages the posterior cingulate/precuneus. Although the role of color information in the recognition of non-objects is not as clear as the role that color plays in object recognition, we suggest that color information for non-objects induces processes related to visual imagery and mental image representations, perhaps encouraging operations that attempt to associate a non-object with already stored knowledge of familiar objects. Additionally, we did not find any particular brain region that responded only to the naming of $\mathrm{B} \& \mathrm{~W}$ objects, suggesting that the recognition of a $\mathrm{B} \& \mathrm{~W}$ object does not add a cognitive operation to the recognition of a colored object.

\section{ACKNOWLEDGEMENTS}

This work was supported by Fundação para a Ciência e Tecnologia (FCT/POCTI/46955/PSI/2002; IBB/CBME, LA, FEDER/POCI 2010), and a PhD fellowship to Inês Bramão (FCT/SFRH/BD/27381/2006). We also want to thank Paulo Tinoco and Lambertine Tackenberg at Clínica Fernando Sancho, Faro, Portugal, for their help with the FMRI data acquisition.

\section{REFERENCES}

[1] Biederman I. Recognition-by-components: a theory of human image understanding. Psychol Rev 1987; 94: 115-47.

[2] Biederman I, Ju G. Surface versus edge-based determinants of visual recognition. Cogn Psychol 1988; 20: 38-64.

[3] Tanaka J, Weiskopf D, Williams P. The role of color in high-level vision. Trends Cogn Sci 2001; 5: 211-5.

[4] Humphreys G, Goodale MA, Jakobson LS, Servos P. The role of surface information in object recognition: Studies of a visual form agnosic and normal subjects. Perception 1994; 23: 1457-81.

[5] Mapelli D, Behrmann M. The role of color in object recognition: evidence from visual agnosia. Neurocase 1997; 3: 237-47.

[6] Price C, Humphreys G. The effects of surface detail on object categorization and naming. Q J Exp Psychol 1989; 41: 797-827.

[7] Humphreys G, Price C, Riddoch MJ. From objects to names: a cognitive neuroscience approach. Psychol Res 1999; 62: 118-30.

[8] Humphreys G, Riddoch M, Quinland P. Cascade processing in picture identification. Cogn Neuropsychol 1988; 5: 67-103.

[9] Riddoch MJ, Humphreys G. Visual object processing: a cognitive neuropsychological approach. Picture naming. London: Erlbaum UK; 1987; pp. 107-43.

[10] Nagai J-i, Yokosawa K. What regulates the surface color effect in object recognition: color diagnosticity or category? Tech Rep Atten Cogn 2003; 28: 1-4.

[11] Oliva A, Schyns PG. Diagnostic colors mediate scene recognition. Cognit Psychol 2000; 41: 176-210.

[12] Tanaka J, Presnell L. Color diagnosticity in object recognition. Percept Psychophys 1999; 61: 1140-53.
[13] Rossion B, Pourtois G. Revisiting Snodgrass and Vanderwart's object pictorial set: The role of surface detail in basic-level object recognition. Perception 2004; 33: 217-36.

[14] Bartels A, Zeki S. The architecture of the colour centre in the human visual brain: new results and a review. Eur J Neurosci 2000; 12: $172-93$.

[15] Conway B, Moeller S, Tsao D. Specialized color modules in macaque extrastriate cortex. Neuron 2007; 56: 560-73.

[16] Conway B, Tsao D. Color architecture in alert macaque cortex revealed by fMRI. Cereb Cortex 2006; 16: 1604-13.

[17] Lueck CJ, Zeki S, Friston K, Deiber M, Kennerd C, Frackowiak R. The color centre in the cerebral cortex of man. Nature 1989; 340: 386-9.

[18] McKeefry D, Zeki S. The position and topography of the human colour centre as revealed by functional magnetic resonance imaging. Brain 1997; 120: 2229-42.

[19] Murphey D, Yoshor D, Beauchamp M. Perception matches selectivity in the human anterior color center. Curr Biol 2008; 18: 216-20.

[20] Zeki S, Watson J, Lueck CJ, Friston K, Kennard C, Frackowiak R. A direct demonstration of functional specialization in human visual cortex. J Neurosci 1991; 11: 641-9.

[21] Barbur J, Spang K. Colour constancy and conscious perception of changes of illuminant. Neuropsychology 2008; 46: 853-63.

[22] Beauchamp M, Haxby J, Jennings J, DeYoe E. An fMRI version of the Farnsworth-Munsell 100-hue test reveals multiple colorselective areas in human ventral occipitotemporal cortex. Cereb Cortex 1999; 9: 257-63.

[23] Chao L, Martin A. Cortical regions associated with perceiving, naming, and knowing about colors. J Cogn Neurosci 1999; 11: 2535 .

[24] Martin A, Haxby J, Lalonde F, Wiggs C, Ungerleider L. Discrete cortical regions associated with knowledge of color and knowledge of action. Science 1995; 270: 102-5.

[25] Zeki S, Marini L. Three cortical stages of colour processing in the human brain. Brain 1998; 121: 1669-85.

[26] Morita T, Kochiyama T, Okada T, Yonekura Y, Matsumura M, Sadato N. The neural substrates of conscious color perception demonstrated using fMRI. Neuroimage 2004; 21: 1665-73.

[27] Howard R, Ffytche D, Barnes J, et al. The functional anatomy of imagining and perceiving colour. Neuroreport 1998; 9: 1019-23.

[28] Simmons W, Ramjee V, Beauchamp M, McRae K, Martin A, Barsalou L. A common neural substrate for perceiving and knowing about color. Neuropsychology 2007; 45: 2802-10.

[29] Kellenbach M, Brett M, Patterson K. Large, colorful, or noisy? Attribute- and modality-specific activations during retrieval of perceptual attribute knowledge. Cogn Affect Behav Neurosci 2001; 1: 207-21.

[30] Snodgrass JG, Vanderwart M. A standardized set of 260 pictures: norms for name agreement, image agreement, familiarity, and visual complexity. J Exp Psychol Learn Mem Cogn 1980; 6: 174-215.

[31] Ventura P. Normas para figuras do corpus de Snodgrass e Vanderwart (1980). Lab Psicol 2003; 1: 5-19.

[32] Talairach J, Tournoux P. Co-planar Stereotaxic Atlas of the Human Brain: An Approach to Cerebral Imaging. New York: Thieme Medical Publishers 1998.

[33] Friston K, Holmes A, Worsley K, Poline JB, Frith C, Frackowiak R. Statistical parametric maps in functional imaging: a general linear approach. Hum Brain Mapp 1995; 2: 189-210.

[34] Worsley KJ, Marrett S, Neelin P, Vandal AC, Friston KJ, Evans AC. A unified statistical approach for determining significant signals in images of cerebral activation. Hum Brain Mapp 1996; 4: 5873.

[35] Genovese CL, Lazar NA, Nichols T. Thresholding of statistical maps in functional neuroimaging using the false discovery rate. Neuroimage 2002; 15: 870-8.

[36] Farah M, Aguirre G. Imaging visual recognition: PET and fMRI studies of the functional anatomy of human visual recognition. Trends Cogn Sci 1999; 3: 179-86.

[37] Grill-Spector K. The neural basis of object perception. Curr Opin Neurobiol 2003; 13: 159-66.

[38] Grill-Spector K, Sayres R. Object recognition insights from advances in fMRI methods. Curr Direct Psychol Sci 2008; 17: 73-9.

[39] Moore C, Price C. Three distinct ventral occipitotemporal regions for reading and object naming. Neuroimage 1999; 10: 181-92.

[40] Price C, Devlin J, Moore C, Morton C, Laird A. Meta-analyses of object naming: Effect of Baseline. Hum Brain Mapp 2005; 25: 7082 . 
[41] Stiers P, Peeters R, Lagae L, Hecke P, Sunaert S. Mapping multiple visual areas in the human brain with a short fMRI sequence. Neuroimage 2006; 29: 74-89.

[42] Vihla M, Laine M, Salmelina R. Cortical dynamics of visual/semantic vs. phonological analysis in picture confrontation. Neuroimage 2006; 33: 732-8.

[43] Bar M, Tootell R, Schacter D, et al. Cortical mechanisms specific to explicit visual object recognition. Neuron 2001; 29: 529-35.

[44] Etard O, Mellet E, Papathanassiou D, et al. Picture naming without Broca's and Wernicke's area. Neuroreport 2000; 11: 617-22.

[45] Wiggs C, Weisberg J, Martin A. Neural correlates of semantic and episodic memory retrieval. Neuropsychology 1999; 37: 103-18.

[46] Pulvermüller F, Hauk O. Category-specific conceptual processing of color and form in left fronto-temporal cortex. Cereb Cortex 2006; 16: 1193-201.

[47] Ueno A, Abe N, Suzuki M, et al. Reactivation of medial temporal lobe and occipital lobe during the retrieval of color information: A positron emission tomography study. Neuroimage 2007; 34: 12928.

[48] Chao L, Haxby J, Martin A. Attribute-based neural substrates in temporal cortex for perceiving and knowing about objects. Nat Neurosci 1999; 2: 913-9.

[49] Demonet JF, Wise R, Frackowiak R. Language functions explored in normal subjects by positron emission tomography: a critical review. Hum Brain Mapp 1993; 1: 39-47.

[50] Ganis G, Schendan H, Kosslyn S. Neuroimaging evidence for object model verification theory: role of prefrontal control in visual object categorization. Neuroimage 2007; 34: 384-98.
[51] Murtha S, Chertkow H, Beauregard M, Evans A. The neural substrate of picture naming. J Cogn Neurosci 1999; 11: 399-423.

[52] Petersen SE, Fox PT, Posner MI, Mintun M, Raichle ME. Positron emission tomographic studies of the cortical anatomy of singleword processing. Nature 1988; 331: 585-9.

[53] Vandenberghe R, Price C, Wise R, Josephs O, Frackowiak RSJ. Functional anatomy of a common semantic system for words and pictures. Nature 1996; 383: 254-6.

[54] Kosslyn SM, Alpert NM, Thompson WL, Chabris CF, Rauch SL Anderson AK. Identifying objects seen from different viewpoints: a PET investigation. Brain 1994; 117: 1055-71.

[55] Oliver R, Thompson-Schill S. Dorsal stream activation during retrieval of object size and shape. Cogn Affect Behav Neurosci 2003; 3: 309-22.

[56] Corbetta M, Patel G, Shulman G. The reorienting system of the human brain: From environment to theory of mind. Neuron 2008; 58: 306-24.

[57] Gerlach C, Law I, Gade A, Paulson OB. Perceptual differentiation and category effects in normal object recognition. Brain 1999; 122: 2159-70.

[58] Martin A, Wiggs C, Ungerleider L, Haxby J. Neural correlates of category-specific knowledge. Nature 1996; 379: 649-52.

[59] Moore C, Price C. A functional neuroimaging study of the variables that generate category-specific object processing differences. Brain 1999; 122: 943-62.

[60] Joseph JE. Functional neuroimaging studies of category specificity in object recognition: A critical review and meta-analysis. Cogn Affect Behav Neurosci 2001; 1: 119-36.

(C) Petersson et al.; Licensee Bentham Open.

This is an open access article licensed under the terms of the Creative Commons Attribution Non-Commercial License (http://creativecommons.org/licenses/by-nc/3.0/) which permits unrestricted, non-commercial use, distribution and reproduction in any medium, provided the work is properly cited. 